\title{
OsCAF2 contains two CRM domains and is necessary for chloroplast development in rice
}

Lan Shen ${ }^{1+}$, Qiang Zhang ${ }^{1+}$, Zhongwei Wang², Hongling Wen', Guanglian Hu', Deyong Ren', Jiang Hu', Li Zhu', Zhenyu Gao', Guangheng Zhang ${ }^{1}$, Longbiao Guo', Dali Zeng ${ }^{1}$ and Qian Qian ${ }^{1 *}$ (I)

\begin{abstract}
Background: Chloroplasts play an important role in plant growth and development. The chloroplast genome contains approximately twenty group II introns that are spliced due to proteins encoded by nuclear genes. CAF2 is one of these splicing factors that has been shown to splice group IIB introns in maize and Arabidopsis thaliana. However, the research of the OsCAF2 gene in rice is very little, and the effects of OsCAF2 genes on chloroplasts development are not well characterized.

Results: In this study, oscaf2 mutants were obtained by editing the OsCAF2 gene in the Nipponbare variety of rice. Phenotypic analysis showed that mutations to OsCAF2 led to albino leaves at the seeding stage that eventually caused plant death, and oscaf2 mutant plants had fewer chloroplasts and damaged chloroplast structure. We speculated that OsCAF2 might participate in the splicing of group IIA and IIB introns, which differs from its orthologs in A. thaliana and maize. Through yeast two-hybrid experiments, we found that the C-terminal region of OsCAF2 interacted with OsCRS2 and formed an OsCAF2-OsCRS2 complex. In addition, the N-terminal region of OsCAF2 interacted with itself to form homodimers.
\end{abstract}

Conclusion: Taken together, this study improved our understanding of the OsCAF2 protein, and revealed additional information about the molecular mechanism of OsCAF2 in regulating of chloroplast development in rice.

Keywords: OsCAF2, CRM domain, Intron splicing, Chloroplast development, Rice

\section{Background}

Chloroplasts are necessary organelles for plant growth and development, as they biosynthesize carbohydrates through the fixation of $\mathrm{CO}_{2}[1,2]$. Chloroplasts are derived from proplastids and are semiautonomous organelles [3]. The chloroplast genome is small and encodes approximately 100 proteins responsible largely for photosynthesis, carbon metabolism, and fatty acid synthesis [4]. Many studies have indicated that plastid-

\footnotetext{
* Correspondence: qianqian188@hotmail.com

${ }^{\dagger}$ Lan Shen and Qiang Zhang contributed equally to this work.

'State Key Laboratory of Rice Biology / China National Rice Research Institute, Chinese Academy of Agricultural Sciences, Hangzhou 310006, China Full list of author information is available at the end of the article
}

encoded polymerases (PEPs) and nucleus-encoded polymerases (NEPs) play key roles in regulating chloroplast development. These studies suggest that expressions of chloroplast genes require proteins encoded by the nuclear genome $[5,6]$. These proteins are involved in the transcription and post-transcription modification of chloroplast related genes, such as chloroplast DNA replication, RNA transcription, RNA editing, RNA stability, and RNA splicing [6].

The process of RNA splicing occurs by removing introns from the precursor messenger mRNA (pre-mRNA) to join adjacent exons [7]. In plants, the introns of chloroplast genes are divided into two groups, known as

C C The Author(s). 2020 Open Access This article is licensed under a Creative Commons Attribution 4.0 International License, which permits use, sharing, adaptation, distribution and reproduction in any medium or format, as long as you give appropriate credit to the original author(s) and the source, provide a link to the Creative Commons licence, and indicate if changes were made. The images or other third party material in this article are included in the article's Creative Commons licence, unless indicated otherwise in a credit line to the material. If material is not included in the article's Creative Commons licence and your intended use is not permitted by statutory regulation or exceeds the permitted use, you will need to obtain permission directly from the copyright holder. To view a copy of this licence, visit http://creativecommons.org/licenses/by/4.0/. The Creative Commons Public Domain Dedication waiver (http://creativecommons.org/publicdomain/zero/1.0/) applies to the data made available in this article, unless otherwise stated in a credit line to the data. 
group I and group II introns, based on their conserved primary and secondary structures, as well as the splicing mechanism of RNA splicing. Group II introns are further divided into two subgroups, subgroup IIA and subgroup IIB [7, 8]. Previous work has shown that group I and group II introns of chloroplast genes have lost the ability for autocatalytic splicing, and the introns of these genes lose elements that were necessary for RNA-based catalytic centers $[8,9]$. Chloroplast RNA splicing requires the recruitment of proteins that are encoded by nuclear genes. These proteins act as splicing factors that are essential for chloroplast RNA splicing reactions [7, 10]. In Arabidopsis thaliana, chloroplast genome contains 20 group II introns and 1 group I intron. In maize and rice, the chloroplast genome consists of 17 group II introns and one group I intron [11]. It has been reported that many nuclear genes encode proteins that act as splicing factors that are involved in the regulation of chloroplast RNA splicing. For example, pentatricopeptide repeat (PPR) proteins and chloroplast RNA splicing and ribosome maturation (CRM) domain proteins are involved in the regulation of splicing of chloroplast gene introns [7].

Previous studies also showed that many PPR proteins participate in the splicing of group II introns in various plant species [12, 13]. For example, in maize, ZmPPR4 and ZmPPR5 promote intron splicing of rps 12 and $\operatorname{trn} G$ in the chloroplast $[14,15]$. In A. thaliana, AtOTP70 and AtOTP51 are required for intron splicing of $r p o C 1$ and $y c f 3-2[16,17]$. In rice, WSL4 and PGL12 participate in intron splicing of $r p l 2$ and $n d h A$, respectively $[18,19]$. Inactivation of the PPR proteins involved in chloroplast intron splicing leads to abnormal chloroplast development, with leaves showing chlorosis or albino seedling phenotypes [20, 21].

With the exception of PPR proteins, some CRM domain-containing proteins act as splicing factors that regulate the splicing of chloroplast introns. It has been reported that the CRM domain-containing proteins influence chloroplast development in A. thaliana, maize, and rice [7]. The first CRM gene, CRS1, was identified and cloned in maize. ZmCRS1 contains three CRM domains, which have high affinity and specificity in vitro for the $a t p F$ intron, and they participate in intron splicing of atpF $[9,22]$. ZmCAF1 and ZmCAF2 contain two CRM domains, and each of them can interact with $\mathrm{ZmCRS} 2$ and form ZmCAF1-ZmCRS2 and ZmCAF2-ZmCRS2 complexes [23]. The ZmCAF1-ZmCRS2 complex is required for the splicing of subgroup IIB introns, including rpl16, rps16, trnG, petD, and $y c f 3$ [24]. In A. thaliana, the AtCAF1-AtCRS2 complex also promoted the splicing of the $r p o C 1$ and $C l p P$ introns, which were absent in the maize chloroplast genome [25]. Furthermore, earlier studies showed that the $\mathrm{C}$-terminal region of $\mathrm{ZmCAF2}$ can interact with ZmCRS2 to form the ZmCAF2-ZmCRS2 complex, which was required for splicing of five subgroup IIB introns from the genes $n d h A, n d h B, \operatorname{rps} 12$, petB, and $y c f 3[23,24]$. The CFM2 has four CRM domains. In maize, ZmCFM2 was required to splice the $\operatorname{trn} L, n d h A$, and $y c f 3$ introns [26]. In A. thaliana, AtCFM2 can splice the $\operatorname{trn} L$, $n d h A$, and $y c f 3$ introns, and it influences splicing of the clpP intron that was absent from the maize chloroplast genome [26]. Unlike other proteins contained in the CRM domains, CFM3 contains three CRM domains and was located in both chloroplasts and mitochondria. In A. thaliana, AtCFM3a was necessary for splicing of the $n d h B$ intron [26]. In rice, fourteen proteins containing one or more CRM domains have been identified [7]. The oscfm3 mutant resulted in an albino phenotype in seedlings caused by abnormal chloroplast development. OsCFM3 promotes splicing of chloroplast genes $n d h B$, petD, and rps16 [26]. Previous research showed that $A L 2$ encodes OsCRS1, which is homologous to AtCRS1 and ZmCRS1, and contains three CRM domains. However, OsCRS1 promotes splicing of group I $(\operatorname{trn} L)$ and group II introns (atpF, $r p l 2, n d h A, n d h B$, petD, and $y c f 3$ ) that differ from the homologous proteins AtCRS1 and ZmCRS1 [11]. It has been reported that OsCAF1 contains two CRM domains that could interact with OsCRS2 via its C-terminal region and forms the OsCAF1-OsCRS2 complex in rice. The OsCAF1 localizes to the chloroplast and affects splicing of subgroup IIA and subgroup IIB introns [27]. The function of other proteins containing the CRM domain has not been studied extensively in rice.

In this study, we generated an oscaf 2 mutant by using the CRISPR/Cas9 (Clustered Regularly Interspaced Short Palindromic Repeats) gene-editing system and studied the role of OsCAF2 in rice chloroplast development. Our results showed that mutants of OsCAF2 lead to abnormal chloroplast development and albino phenotypes in seedlings. Analysis of intron splicing of chloroplast genes showed that OsCAF2 promoted the splicing of chloroplast subgroup IIA and subgroup IIB introns, which were necessary for chloroplast development. Similar to the ZmCAF2-ZmCRS2 complex, the C-terminal region of OsCAF2 interacted with OsCRS2 and formed the OsCAF2-OsCRS2 complex. Interestingly, OsCAF2 also could interact with itself through its $\mathrm{N}$-terminal region to form dimers that regulate chloroplast development in rice. These results demonstrate the molecular mechanism of OsCAF2 in rice chloroplast development.

\section{Results}

\section{Disruption of OsCAF2 function caused an albino phenotype}

According to previous studies, the gene homologous to $Z m C A F 2$ and $A t C A F 2$ from maize and A. thaliana was identified from the rice genome and named OsCAF2 ( $L O C_{-}$ Os01g21990) [7]. The OsCAF2 contains six exons, with a 
total coding region of $1824 \mathrm{bp}$, which encodes 608 amino acids. The OsCAF2 contains two CRM domains, but their functions are unknown. To study the function of OsCAF2, we constructed the OsCAF2 gene editing vector including two target sgRNAs in exon 1 (upstream of the two CRM domains) and exon 3 (the first CRM domain) respectively, and transformed it into Nipponbare via the Agrobacterium method (Fig. 1a-b). In the $\mathrm{T}_{0}$ generation, we obtained 15 plants. Through PCR amplification and sequencing of OsCAF2, we screened one homozygous mutants and one heterozygote plant, named oscaf2 and oscaf2/OsCAF2, respectively (Fig. 1c). The mutation types of one or two base insertions lead to the premature termination of OsCAF2 translation. Phenotypic observations suggested that the oscaf 2 mutants showed albino seedling phenotypes and died at the three leaf stage, but the heterozygote oscaf2/OsCAF2 showed green leaves and normal growth (Fig. 1d). To investigate the function of OsCAF2, we obtained oscaf2 mutants in the $\mathrm{T}_{1}$ generation of oscaf2/OsCAF2 heterozygote plant (Fig. 2a). The oscaf2 mutants from oscaf2/OsCAF2 heterozygote plant also appeared as albino and then died at the three leaf stage (Fig. 2a). Compared to wild type (WT), the Chl a, $\mathrm{Chl} \mathrm{b}$, car and Chl content in oscaf2 mutant leaves were significantly decreased, and was practically devoid of car and Chl b (Fig. 2b). These results suggest that OsCAF2 was necessary for chloroplast development, and that mutation of OsCAF2 resulted in an albino phenotype in seedlings.
Expression analysis of chloroplast development and photosynthesis-related genes in oscaf2 mutants

To further analysis the effects of impaired chloroplast development in the oscaf 2 mutant, we measured the expression levels of chloroplast development and photosynthesis-related genes. PEPs and NEPs are regulated transcriptionally by chloroplast-encoded genes responsible for chloroplast development. Expression levels of the NEP component gene OsRpoTp in the oscaf2 mutant was normal compared to the WT (Fig. 2c). However, except the gene $r p o A$, there were significant increases in the expression levels of PEP component genes, including rpoB, rpoC1, and rpoC2, in the oscaf2 mutant relative to WT (Fig. 2c). By contrast, the expressions of photosynthesis-related genes involving $R b c S$, RbcL, HEMA, CHLI, psbO, HEMD, psbA, and CHLM were dramatically reduced in the oscaf2 mutant (Fig. 2c). These results suggest that OsCAF2 might affect the expression level of chloroplast development and photosynthesis-related genes.

\section{Chloroplast development was defected in oscaf2 mutant}

To further study the albino leaf phenotype in the oscaf2 mutant, we observed the ultrastructure of chloroplasts of leaves in WT and oscaf2 in seedling stage using transmission electron microscopy (TEM). Our result showed that the integrated chloroplast structures and normal grana stacks were found in WT leaves (Fig. 2d-e), and

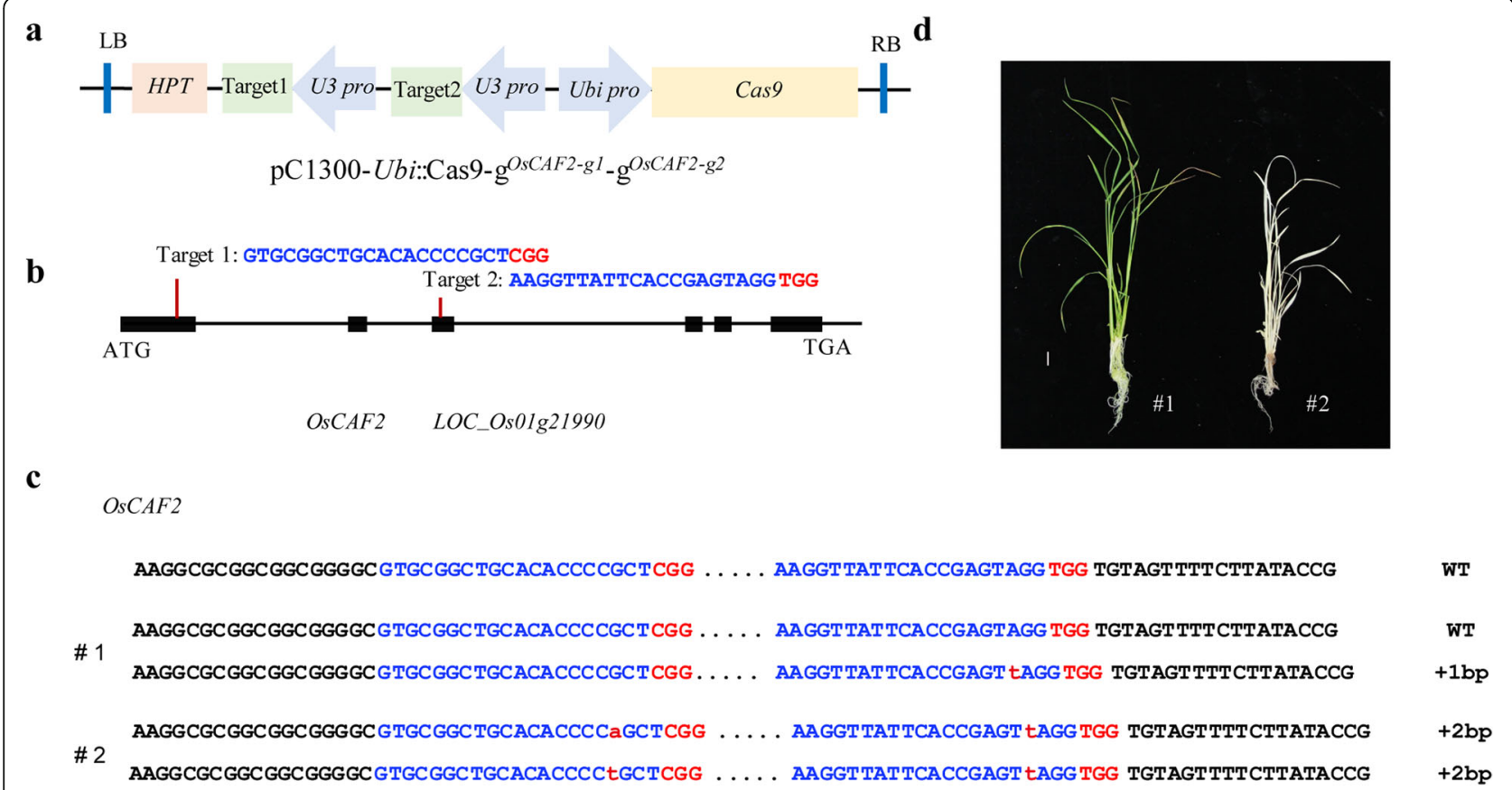

Fig. 1 Disruption of OsCAF2 results in an albino phenotype. a Schematic diagram of CRISPR/Cas9 system for editing OsCAF2. b Diagram of the targeted site in OsCAF2. c Mutation types of transformed plants in the $T_{0}$ generation. The protospacer adjacent motif sequence is shown in red. The targeted sequences are shown in blue. $\mathbf{d}$ The phenotype of the two positive plants at the seedling stage. Scale bar $=1 \mathrm{~cm}$ 
a
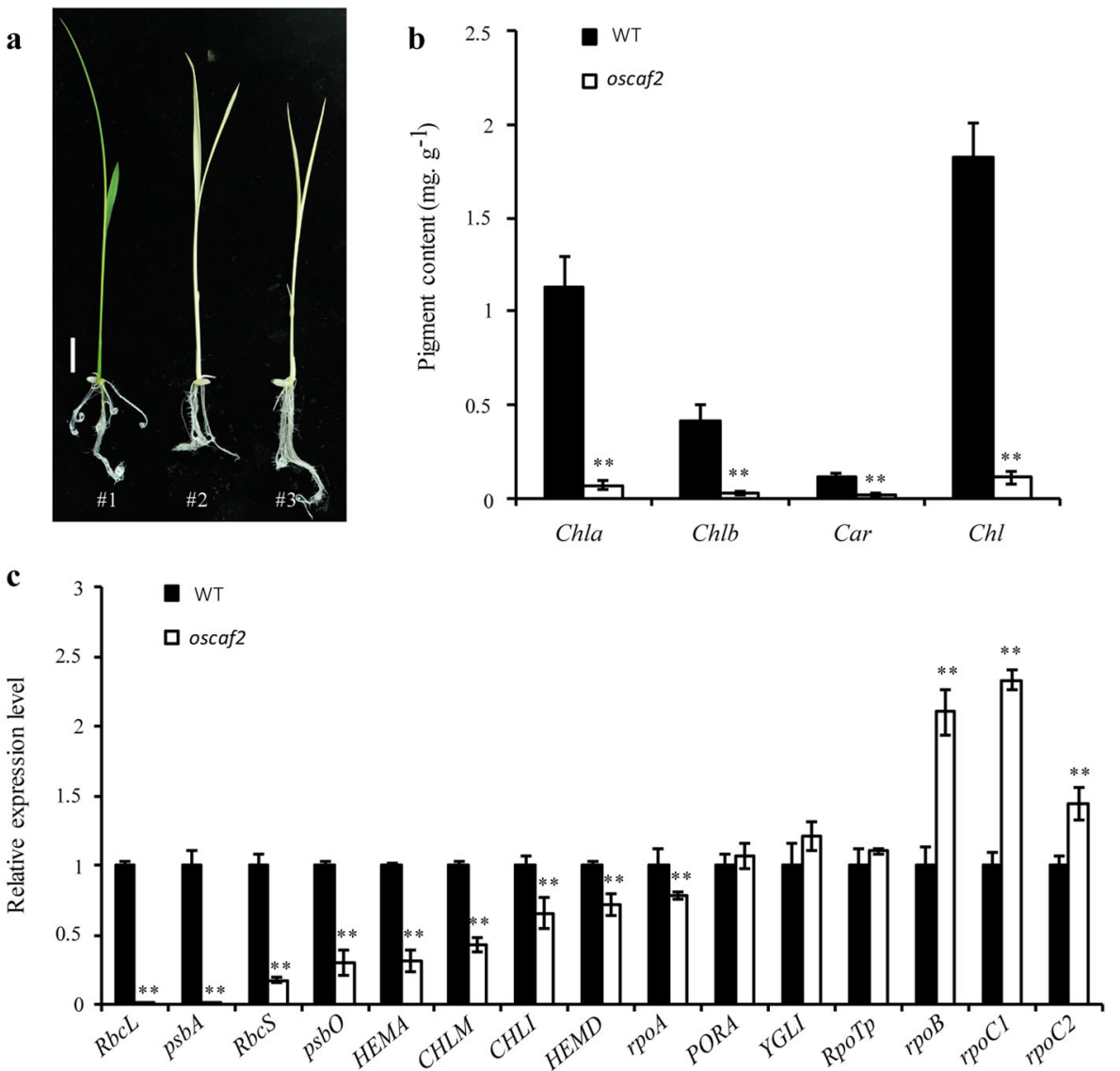
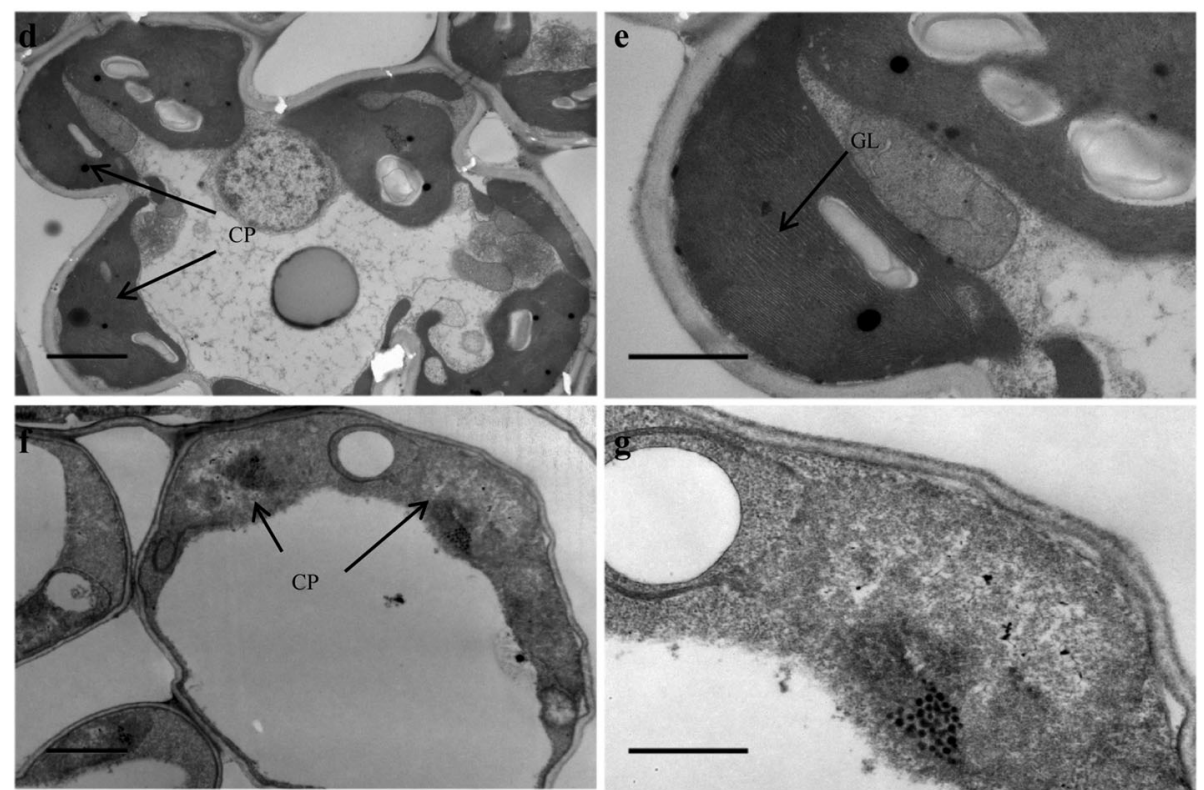

Fig. 2 Chloroplast development was impaired in the oscaf2 mutant. a Phenotype presentation of WT (\#1) and oscaf2 mutant (\#2 and \#3). Scale bar $=1 \mathrm{~cm}$. $\mathbf{b}$ Chlorophyll contents of WT and oscaf2 at the two leaf stage. $\mathbf{c}$ Expression analysis of chloroplast synthesis and photosynthesisrelated genes in WT and the oscaf2 mutant. The values represent the mean of three independent experiments. Error bars indicate $\mathrm{SE}$, ** indicate $P<0.01$, respectively. $\mathbf{d}-\mathbf{g}$ The chloroplast ultrastructure of $W T$ and oscaf2 mutants from leaves. WT (d, e) and oscaf2 mutants (f, $\mathbf{g})$. CP, chloroplast, GL, grana lamella. Scale bars of $\mathbf{d}$ and $\mathbf{f}, 2 \mu \mathrm{m}$, Scale bars of $\mathbf{e}$ and $\mathbf{g}, 1 \mu \mathrm{m}$ 
the abnormal chloroplast structure and the disorganized grana lamellar stacks in oscaf2 mutants (Fig. $2 \mathrm{f}-\mathrm{g}$ ). These data suggest that the mutation to OsCAF2 impaired chloroplasts development in rice. These results also suggest that OsCAF2 is necessary for chloroplast development.

\section{OsCAF2 influences splicing of group II introns}

In $A$. thaliana and maize, CAF2 promoted the splicing of the chloroplast RNA group IIB introns [25]. Therefore, we examined whether OsCAF2 was involved in chloroplast RNA intron splicing in rice. The rice chloroplast genome contains 17 group II introns and 1 group I intron. We amplified chloroplast genes that contained at least one intron using RT-PCR and compared intron splicing efficiency between the WT and oscaf2 mutant. We found abnormal intron splicing of subgroup IIA introns (rpl2, rps12, and $a t p F)$ and subgroup IIB introns ( $n d h A, n d h B$, and $y c f 3$ ) in the oscaf2 mutant, compared with the WTs (Fig. 3, Fig. S1). However, the intron splicing of trnL (group I) was not affected in the oscaf2 mutant (Fig. 3, Fig. S1). These results suggested that OsCAF2 might participate in splicing of subgroup IIA and subgroup IIB introns but not group I introns.

\section{Expression patterns and subcellular localization of OsCAF2}

According to the gene expression and protein tools of rice (http://www.bar.utoronto.ca/efprice/cgi-bin/efpWeb. cgi), OsCAF2 was highly expressed in leaves but low or undetectable level was found in spikes and root. To validate the result, we analyzed the expression pattern of OsCAF2 in different tissues at the heading stage of Nipponbare using qRT-PCR. Compared with the root, the expression level of other tissues were significantly increased, and the gene expression in leaves even more than a thousand times (Fig. 4a). Our results indicated that $O s C A F 2$ expression was highest in green tissues, especially in leaves.

The Wolf PSORT (https://wolfpsort.hgc.jp/) website predicted that OsCAF2 was localized to rice chloroplasts. To determine the subcellular localization of OsCAF2, we constructed the OsCAF2-GFP fusion vector and transformed it into rice protoplasts. The p580 empty vector was used as a control. The results showed that the empty vector had green fluorescent signals in the nucleus, membrane, and cytoplasm, but the OsCAF2GFP fusion protein produced signals that co-localized with the chloroplast autofluorescence signals, suggesting

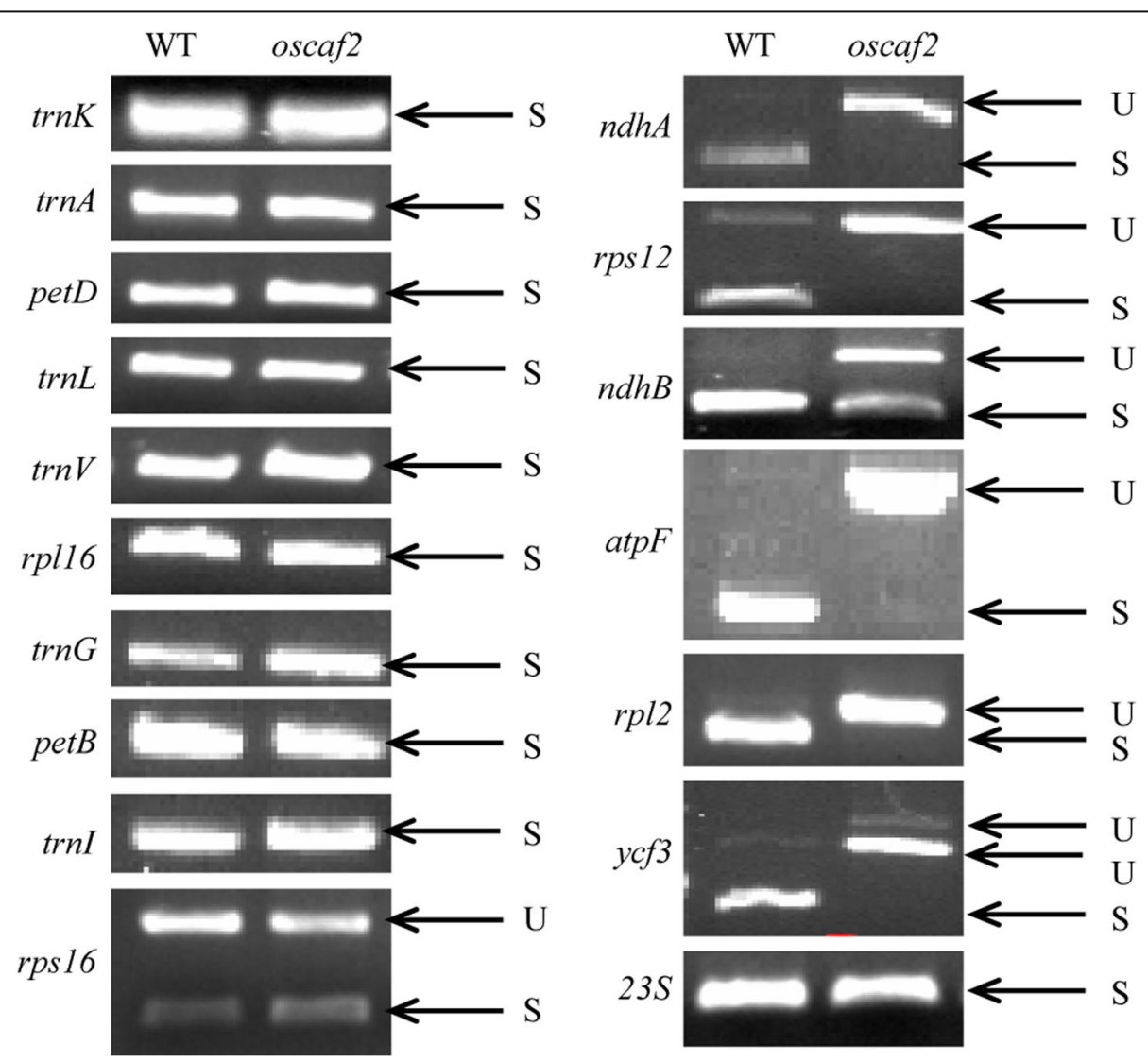

Fig. 3 Splicing analysis of group I and group II introns in WT and oscaf2 mutants. U indicates unspliced transcripts. S indicates spliced transcripts 

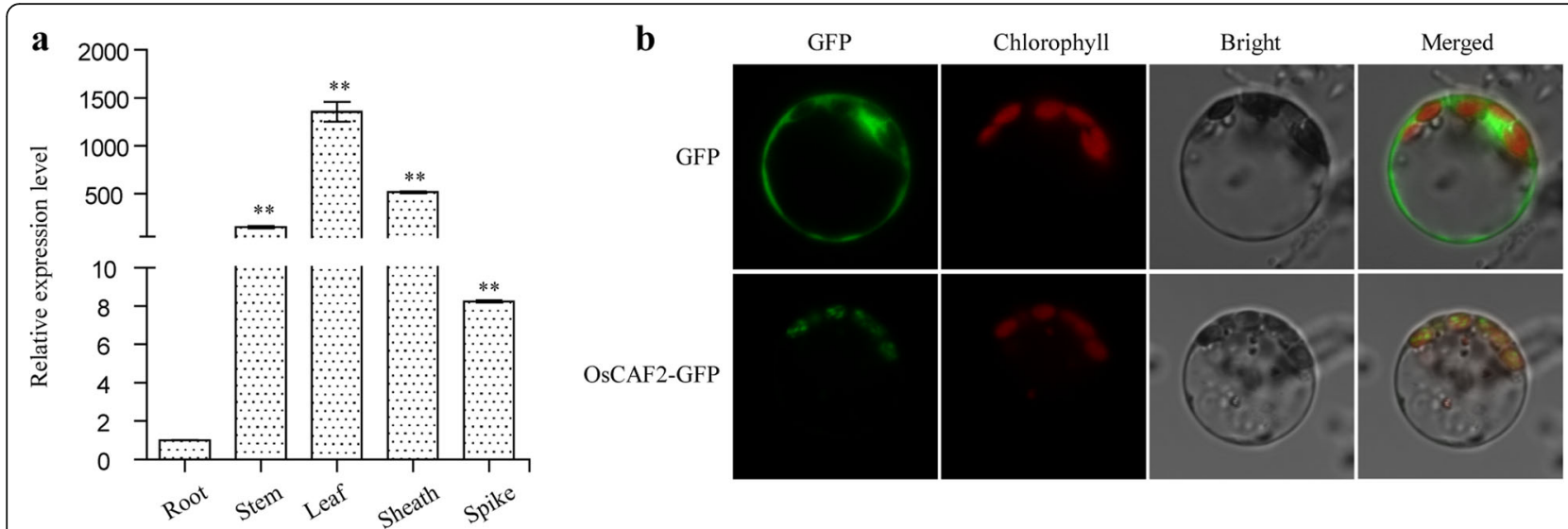

Fig. 4 Expression pattern and subcellular localization of OsCAF2. a Expression pattern of OsCAF2 in various tissues, including root, stem, leaf, sheath, and spike at the heading stage. The values represent the mean of three independent experiments. Error bars indicate $\mathrm{SE},{ }^{* *}$ indicates $P<$ 0.01. b Subcellular localization of OSCAF2 protein in rice protoplasts

that OsCAF2 localizes in chloroplasts (Fig. 4b, Fig. S2). This result supports the view that OsCAF2 plays an important role in regulating chloroplast development.

\section{C-terminal region of OsCAF2 interacts with OsCRS2}

Previous studies show that the N-terminal region of CAF2 interacts with CRS2 and forms the CAF2-CRS2 complex in maize [23]. To confirm the relationship between CAF2 and CRS2 in rice, we acquired the coding sequence (CDS) of OsCRS2 (LOC_Os01g04130) from rice leaves and constructed the OsCRS2-PGADT7 (OsCRS2-AD) and OsCAF2-PGBKT7 (OsCAF2-BD) vectors for yeast twohybrid $(\mathrm{Y} 2 \mathrm{H})$ analysis. The AH109 yeast cell that contained either OsCRS2-AD and the empty BD vector or OsCAF2$\mathrm{BD}$ and the empty $\mathrm{AD}$ vector were able to grow on synthetic dextrose medium lacking Leu and Trp (SD-T/L), but they were unable to grow on medium lacking Leu, Trp, His, and Ade (SD-L/T/H/A). However, the AH109 yeast cell that contained OsCRS2-AD and OsCAF2-BD, OsCRS2-BD and OsCAF2-AD were able to grow in SD-T/ $\mathrm{L}$ and SD-L/T/H/A (Fig. 5a, Fig. S3). These results suggest that OsCAF2 can interact with OsCRS2 to form the OsCAF2-OsCRS2 complex in rice. We then truncated the OsCAF2 protein into three sections, named OsCAF2-N (N-terminal), OsCAF2-M (middle), and OsCAF2-C (C-terminal) and repeated the $\mathrm{Y} 2 \mathrm{H}$ experiment (Fig. 5b). Among these sections, OsCAF2-M contains the two CRM domains. The OsCAF2-C interacted with OsCRS2 but OsCAF2-N and OsCAF2-M did not (Fig. 5c, Fig. S3). These results indicate that the C-terminal of OsCAF2 is necessary for the interaction with OsCRS2.

\section{OsCAF2 forms homodimers through the $\mathrm{N}$-terminal region}

In order to study whether OsCAF2 could form homodimers, we carried out an $\mathrm{Y} 2 \mathrm{H}$ analyzes. In brief, we constructed OsCAF2-AD and OsCAF2-BD vectors and then co-transformed them into AH109 yeast cells. The $\mathrm{Y} 2 \mathrm{H}$ results showed that OsCAF2 could self-interact and form homodimers (Fig. 6a, Fig. S4). We divided OsCAF2 into the three sections as above, to further analyze which section was responsible for forming homodimers. Only the $\mathrm{N}$-terminal region of OsCAF2 was able to interact with itself, but it could not interact either with the $\mathrm{M}$ or Cterminal regions of OsCAF2 (Fig. 6b, Fig. S4). Therefore, the N-terminal of OsCAF2 was necessary to form homodimers.

\section{Discussion}

The OsCAF2 protein has two CRM domains with homology to AtCAF2 and ZmCAF2. Our phenotypic results of the oscaf2 mutant were consistent with the albino mutant atcaf2 in A. thaliana, which was obtained by TDNA insertion [25]. Therefore, we suspected that the function of CAF2 might be highly conserved in A. thaliana and rice. In rice, $A L 2$, OsCAF1, and CFM3 encoding proteins contained CRM domains, and the al2, oscaf1, and oscfm 3 mutants showed albino leaf phenotypes with disrupted chloroplast structure [11, 26, 27]. Research shows there were 14 proteins containing one or more CRM domains, and four of them contained two CRM domains in rice [7]. As the albino phenotypic of oscaf2 mutants, we speculated that the function of OsCAF2 was not redundant with the other three proteins that contain two CRM domains. These results suggest that CRM domain proteins play a key role in chloroplast development in rice.

It has been reported that CAF2 is a splicing factor that is encoded by nuclear genes that participate in intron splicing of petB, rps12, ndhA, and $y c f 3$ in maize and $A$. thaliana [25]. This indicates that the molecular mechanism of CAF2 regulation of chloroplast development is 


\section{$\mathbf{a}$}

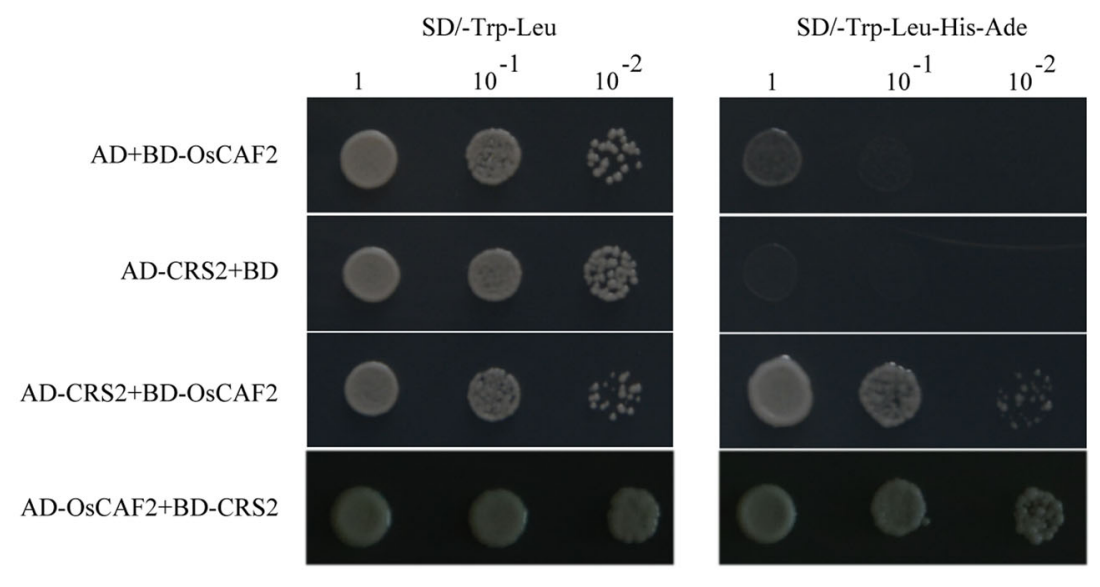

b

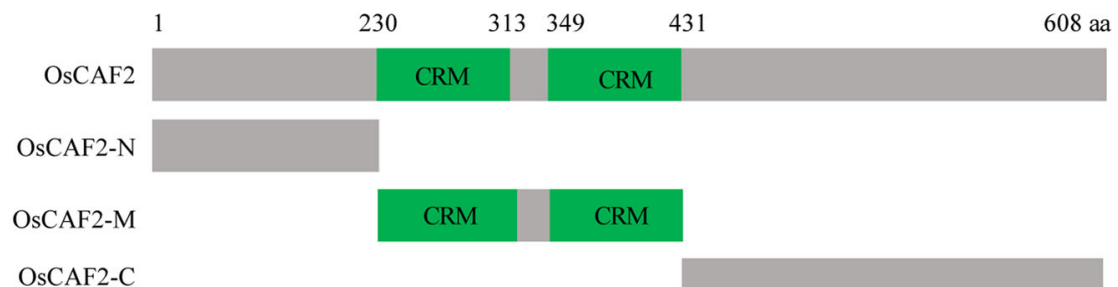

C

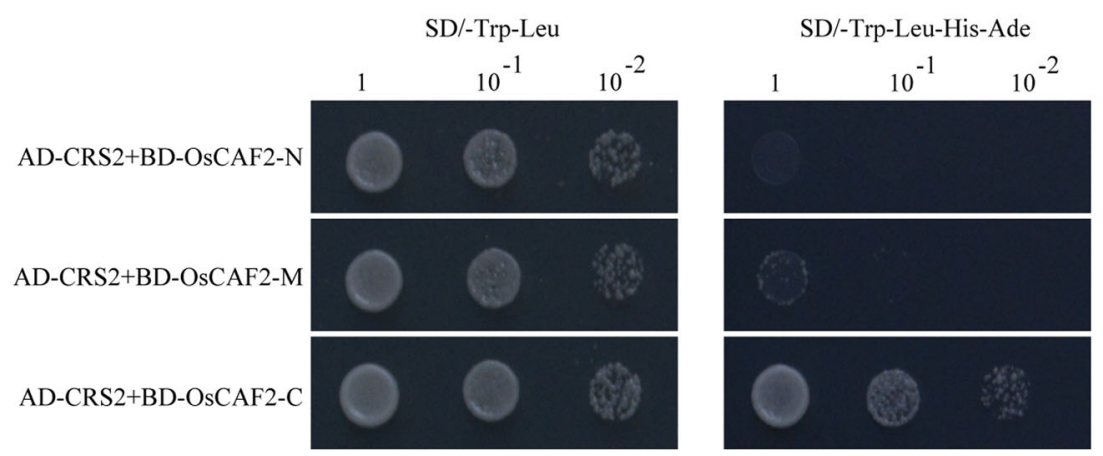

Fig. 5 OsCAF2 interacts with OsCRS2 through the C-terminal region. a The Y2H interaction between OsCAF2 and OsCRS2. The full-length CDS of OsCAF2 and OsCRS2 were cloned into pGBKT7 (BD) and PGADT7 (AD) vectors to produce OsCAF2-BD, OsCAF2-AD, OsCRS2-BD, and OsCRS2-AD plasmids, respectively. The OsCAF2-BD and OsCRS2-AD, OsCAF2-AD and OsCRS2-BD were co-transformed into the AH109 yeast strain. The OsCAF2-BD and AD plasmid, and OsCRS2-AD and BD plasmid were co-transformed into the AH109 yeast strain as a control. b Schematic diagram of OsCAF2 protein structure. Different sections of OsCAF2 involving in OsCAF2-N (1-230 aa), OsCAF2-M (231-430 aa), and OsCAF2-C (431-608 aa). c $\mathrm{Y} 2 \mathrm{H}$ interaction of OsCRS2 and different truncated sections of OsCAF2. The truncated sections of OsCAF2 were cloned into the BD vector to generate OsCAF2-N-BD, OsCAF2-M-BD, and OsCAF2-C-BD vectors. These three vectors were co-transferred into AH109 yeast cells with the OsCRS2-AD vector. The yeast cells were cultured on SD-T/L and SD-/T/L/H/A media with different dilution series $\left(1,10^{-1}\right.$, and $\left.10^{-2}\right)$

relatively conserved in maize and $A$. thaliana. In this study, by analyzing the intron splicing of chloroplast genes, we found that OsCAF2 promotes the splicing of subgroup IIB and subgroup IIA introns. For example, OsCAF2 influences $r p l 2$ and atpF intron splicing, but ZmCAF2 and AtCAF2 does not influence splicing of these two introns in maize and A. thaliana. However, OsCAF2, AtCAF2, and ZmCAF2 all affect splicing of $n d h A$ and $y c f 3$ introns. Therefore, this suggests that OsCAF2 has a conserved but distinct role compared to
AtCAF2 and ZmCAF2. Similar to OsCAF1 and AL2 in rice, OsCAF2 affects splicing of subgroup IIA and subgroup IIB introns $[11,27]$. The molecular mechanism of OsCAF2 regulating chloroplast development may be different from AtCAF2 and ZmCAF2. The function of OsCAF2 in splicing of subgroup IIA introns, such as atpF and $r p l 2$ introns, requires further study.

In plants, chloroplast development is mainly divided into three steps [28]. NEP and PEP participate in the second and third steps to regulate the transcription of 


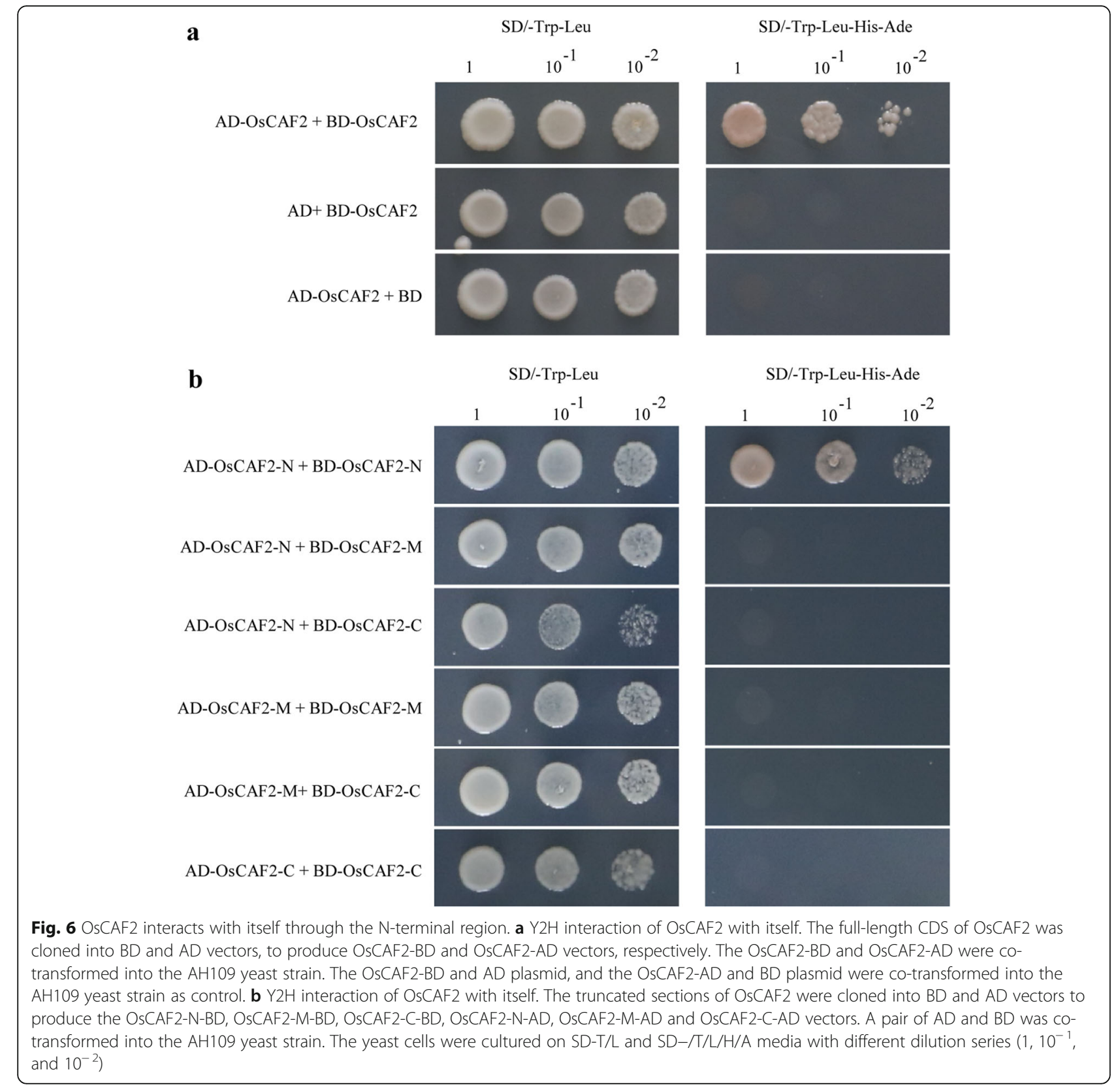

plastid genes [28]. In the oscaf2 mutant, the expression levels of the NEP component (OsRpoTp) are similar to the WT, while the expression of PEP components $(r p o B$, rpoC1, and rpoC2) increased. This might be caused by a feedback regulation mechanism: impaired chloroplast development might induce transcription of genes with PEP components. Similar conclusions were acquired in many chloroplast defective mutants, such as asl3 and sal4, in rice. Previous work showed that asl3 and sal4 also exhibited albino leaf phenotypes and expression levels of rpoB, rpoC1, and rpoC2 in asl3 and sal4 increased significantly $[18,29]$. By contrast, expression levels of photosynthesis-related genes, such as $R b c S$,
RbcL, HEMA, CHLI, psbO, HEMD, psbA, and CHLM decreased. It may be possible that the signals from the plasmid to the nucleus might be different in the oscaf 2 mutant. This result of signal transduction was also consistent with previous results in the osptac2 mutant [30]. Thus, these results showed that OsCAF2 might influence chloroplast development through its effects on PEP and photosynthesis-related gene expression.

It was reported that CAF2 interacted with CRS2 through its C-terminal region in maize [23]. CRS2 was a group II intron splicing factor that could promote the splicing of nine group II introns [31, 32]. Gene expression analysis showed that expression levels of OsCAF2 
were highest in leaves. The expression pattern of OsCAF2 was similar to OsCRS2. In this study, we determined that OsCAF2 interacts with OsCRS2 through its $\mathrm{C}$-terminal region. Previous analysis of the C-terminal region of CAF2 in monocot and dicot plants, including Arabidopsis thaliana, maize and rice, found that there was a conservative residue of CAF2 that formed a hydrophobic region necessary for protein interactions with CRS2 [23]. In rice, OsCAF2 might interact with OsCRS2 through this conservative residue. The molecular mechanism of the CAF2-CRS2 complex formation might be conserved across plants. It has been reported that the homodimers and heterodimers might have biological advantages than the individual protein, which could promote the synthesis of products or regulate the activity of partner enzyme domains [33]. Consider the homodimers of OsCAF2 formed via its N-terminal region, we suspected that proper chloroplast development requires recruitment of OsCAF2 and OsCRS2 to form the OsCAF2-OsCRS2 complex, as well homodimers of OsCAF2 to achieve splicing of group II introns. Previous studies have shown that OsCAF1 also contains two CRM domains that could affect splicing of subgroup IIA and subgroup IIB introns, and the orthologous genes of AtCAF1 and ZmCAF1 in A. thaliana and maize only promoted the splicing of subgroup IIB introns [27]. In addition, OsCAF1 interacts with itself to form homodimers. In maize, ZmCRS1 contains three CRM domains and can form homodimers, but whether CAF2 forms homodimers has not been reported in maize and $A$. thaliana [22]. Therefore, this study provides new clues to the function of the CAF2 protein in different plants and provides important information for exploring the development of chloroplasts in rice.

\section{Conclusions}

The present study generated albino lethal oscaf 2 mutants using CRISPR/Cas9 gene-editing system. The following study showed that OsCAF2 could affect chloroplast development, and might participate in the splicing of group IIA and IIB introns, which differs from its orthologs in $A$. thaliana and maize. The $\mathrm{Y} 2 \mathrm{H}$ assay showed that the Cterminal region of OsCAF2 interacted with OsCRS2 and formed an OsCAF2-OsCRS2 complex, and the Nterminal region of OsCAF2 interacted with itself to form homodimers. These findings improved our understanding of the OsCAF2 protein, and revealed additional information about the molecular mechanism of OsCAF2 in regulating of chloroplast development in rice.

\section{Methods}

Plant materials and growth conditions

The japonica variety Nipponbare have been conventionally cultivated in Japan. The seeds of Nipponbare were provided by the China National Rice Research Institute (CNRRI) in Hangzhou, Zhejiang Province, China. We designed the two target sites of OsCAF2 (GTGCGGCT GCACACCCCGCTCGG, AAGGTTATTCACCGAG TAGGTGG) and constructed the pC1300-Ubi::Cas9$\mathrm{gX}^{O S C A F 2-\mathrm{g} 1}$ - $\mathrm{gX}^{\mathrm{OSCAF2-g2}}$ vector, according to previouslydescribed method [34]. Vector construction and sequencing related primers are listed in Table S1. This vector were transformed into Nipponbare via Agrobacteriummediated transformation. The OsCAF2 mutation of the positive transgenic plants were identified by the Sanger method and analyzed by the degenerate sequence decoding method [35]. All the transgenic plants we obtained and their offspring were grown in the greenhouse under constant temperature $\left(30^{\circ} \mathrm{C}\right.$ at $16 \mathrm{~h}$ light and $8 \mathrm{~h}$ dark $)$ of CNRRI in Hangzhou, Zhejiang Province, China.

\section{Pigment content measurement}

The Chlorophyll a (Chla), chlorophyll b (Chlb), carotenoid (Car) and total chlorophyll (Chl) content was measured. In brief, we obtained WT and oscaf2 mutant leaves $(0.2 \mathrm{~g})$ at the seedling stage. The leaves were cut and soaked in $5 \mathrm{ml} 95 \%$ ethanol for $48 \mathrm{~h}$ under dark conditions. Spectrophotometric measurements were conducted using a UV-1800PC spectrophotometer (Mapada, China) at $470 \mathrm{~nm}, 649 \mathrm{~nm}$, and $665 \mathrm{~nm}$. According to previous methods [36], we calculated the Chla, Chlb, Car and Chl content in WT and oscaf2 mutant leaves.

\section{Transmission electron microscopy (TEM) assays}

At the three leaf stage, we samples of WT and oscaf2 mutant leaves were cut into $0.1 \mathrm{~cm} \times 0.1 \mathrm{~cm}$ pieces. The samples were fixed in $2.5 \%$ glutaraldehyde for $4 \mathrm{~h}$ in at $4{ }^{\circ} \mathrm{C}(\mathrm{pH} 7.0)$ and then fixed in $1 \% \mathrm{OsO} 4(\mathrm{pH} 7.4)$. The fixed samples were stained with uranyl acetate. After dehydration in an ethanol series, the samples were embedded in acrylic resin. The samples were staining and observed with a Hitachi-7500 (Tokyo, Japan) transmission electron microscope.

\section{RNA extraction and quantitative real-time PCR (qRT-PCR)}

Total RNA was extracted from leaves using RNA extraction kit (Axygen, USA) according to the manufacturer's protocols. First-strand cDNA was synthesized using the ReverTra Ace qPCR RT Kit (TOYOBO, Japan) according to the manufacturer's instructions. The qRT-PCR analysis process was as follows: $5 \mathrm{~min}$ at $94^{\circ} \mathrm{C}$ followed by 40 cycles of $95^{\circ} \mathrm{C}$ for $15 \mathrm{~s}$ and $58^{\circ} \mathrm{C}$ for $50 \mathrm{~s}$. The OsActin1 was used as a reference gene in this study. The relative expression levels were calculated according to the $2^{-\Delta \Delta C T}$ method [37]. All primers for qRT-PCR are shown in Table S1. 


\section{Splicing analysis of group I and group II introns}

We used RT-PCR to perform splicing analysis of group I and group II introns of chloroplast genes from WT and oscaf2 mutants. The RT-PCR procedure was as follows: $94{ }^{\circ} \mathrm{C}$ for $5 \mathrm{~min}$, followed by 30 cycles of $94^{\circ} \mathrm{C}$ for $30 \mathrm{~s}$, $58^{\circ} \mathrm{C}$ for $30 \mathrm{~s}, 72^{\circ} \mathrm{C}$ for $45 \mathrm{~s}$, and a final elongation step at $72{ }^{\circ} \mathrm{C}$ for $10 \mathrm{~min}$. All chloroplast RNA splicing analysis primers are listed in Table S1.

\section{Subcellular localization of OsCAF2}

To confirm subcellular localization of the OsCAF2 protein, the full-length OsCAF2 coding sequence (remove stop code) was amplified and cloned into the pAN580 vector to create the p580-2 $\times$ CaMV35S::OsCAF2-GFP (OsCAF2GFP) plasmid (Table S2). The OsCAF2-GFP vector construction primers are listed in Table S1. The preparation of rice protoplasts and transformation followed previously described methods [38]. The fluorescence of green fluorescent protein (GFP) was observed using confocal laser scanning microscopy (Zeiss LSM 780, Germany).

\section{Yeast two-hybrid $(\mathrm{Y} 2 \mathrm{H})$ assays}

The full-length and truncated coding sequences of $O s C A F 2$, and full-length OsCRS2 coding sequence were amplified via PCR (Table S2). Relevant PCR amplification primers are listed in Table S1. PCR amplification products were cloned into the pGAD-T7 or pGBK-T7 vectors. The $\mathrm{Y} 2 \mathrm{H}$ experiment used the Clontech two-hybrid system, according to the manufacturer's instructions.

\section{Supplementary information}

Supplementary information accompanies this paper at https://doi.org/10. 1186/s12870-020-02593-z.

\section{Additional file 1: Table S1. The primers used in present study. Table} S2. Sequence of OSCAF2 and OSCRS2 genes from Nipponbare.

Additional file 2: Figure S1. Original images for Fig. 3.

Additional file 3: Figure S2. Original images for Fig. 4b.

Additional file 4: Figure S3. Original images for Fig. 5.

Additional file 5: Figure S4. Original images for Fig. 6.

\section{Abbreviations}

PEPs: Plastid-encoded polymerases; NEPs: Nucleus-encoded polymerases; pre-mRNA: Precursor messenger mRNA; PPR: Pentatricopeptide repeat; CRM: Chloroplast RNA splicing and ribosome maturation; CRISPR: Clustered regularly interspaced short palindromic repeats; TEM: Transmission electron microscopy; Y2H: Yeast two-hybrid; qRT-PCR: RNA extraction and Quantitative real-time PCR; GFP: Green fluorescent protein; CDS: Coding sequence

\section{Acknowledgements}

Not applicable.

\section{Authors' contributions}

SL and ZQ designed the study and wrote the original manuscript. ZQ, SL, WZW, WHL, and HGL conducted all the experiments. RDY, HJ, ZL, GZY, ZGH and GLB were responsible for the data analysis ZDL and QQ modified the manuscript. All authors read and approved the manuscript.

\section{Funding}

This research was supported by the National Natural Science Foundation of China (No. 31801333, No. 31701390), and the Central Public-interest Scientific Institution Basal Research Fund of China National Rice Research Institute (2017RG001-4). The funding bodies provided the financial support to this research, including experimental design and implementation, sampling and data analysis. No funder played the role in data collection and analysis and writing the manuscript.

\section{Availability of data and materials}

The datasets supporting the results of this article are available from the corresponding author on reasonable request. Sequence data used during the current study for the CDNA and genomic DNA of OsCAF2 and OsCRS2 are available from the GenBank/EMBL data libraries under accession numbers LOC_Os01g21990, LOC_Os01g04130, respectively, and could also available from the National Center for Biotechnology Information (NCBI) (OsCAF2 Gene ID: 4326994, OsCRS2 Gene ID: 4325801).

Ethics approval and consent to participate

Not applicable.

\section{Consent for publication}

Not applicable.

\section{Competing interests}

The authors declare that they have no competing interests.

\section{Author details}

${ }^{1}$ State Key Laboratory of Rice Biology / China National Rice Research Institute, Chinese Academy of Agricultural Sciences, Hangzhou 310006, China. ${ }^{2}$ Biotechnology Research Center, Chongqing Academy of Agricultural Sciences, Chongqing 401329, China.

Received: 18 March 2020 Accepted: 12 August 2020

Published online: 18 August 2020

References

1. Jarvis P, López-Juez E. Biogenesis and homeostasis of chloroplasts and other plastids. Nat Rev Mol Cell Biol. 2013;14(12):787-802.

2. Li Y, Zhang J, Li L, Gao L, Xu J, Yang M. Structural and comparative analysis of the complete chloroplast genome of Pyrus hopeiensis- "wild plants with a tiny population" -and three other Pyrus species. Int J Mol Sci. 2018;19(10): 3262

3. Bobik K, Burch-Smith TM. Chloroplast signaling within, between and beyond cells. Front Plant Sci. 2015:6:781.

4. Nakai M. New perspectives on chloroplast protein import. Plant Cell Physiol. 2018:59(6):1111-9.

5. Hedtke B, Börner T, Weihe A. Mitochondrial and chloroplast phage-type RNA polymerases in Arabidopsis. Science. 1997:277(5327):809-11.

6. Yu QB, Huang C, Yang ZN. Nuclear-encoded factors associated with the chloroplast transcription machinery of higher plants. Front Plant Sci. 2014;5: 316.

7. De Longevialle AF, Small ID, Lurin C. Nuclearly encoded splicing factors implicated in RNA splicing in higher plant organelles. Mol Plant. 2010;3(4): 691-705

8. Bonen L, Vogel J. The ins and outs of group II introns. Trends Genet. 2001; 17(6):322-31.

9. Till B, Schmitz-Linneweber C, Williams-Carrier R, Barkan A. CRS1 is a novel group II intron splicing factor that was derived from a domain of ancient origin. Rna. 2001;7(9):1227-38.

10. Barkan A, Goldschmidt-Clermont M. Participation of nuclear genes in chloroplast gene expression. Biochimie. 2000;82(6-7):559-72.

11. Liu C, Zhu H, Xing Y, Tan J, Chen X, Zhang J, et al. Albino Leaf 2 is involved in the splicing of chloroplast group I and II introns in rice. J Exp Bot. 2016; 67(18):5339-47.

12. Khrouchtchova A, Monde RA, Barkan A. A short PPR protein required for the splicing of specific group II introns in angiosperm chloroplasts. Rna. 2012; 18(6):1197-209.

13. Rovira AG, Smith AG. PPR proteins - orchestrators of organelle RNA metabolism. Physiol Plant. 2019;166(1):451-9. 
14. Schmitz-Linneweber C, Williams-Carrier RE, Williams-Voelker PM, Kroeger TS, Vichas A, Barkan A. A pentatricopeptide repeat protein facilitates the transsplicing of the maize chloroplast rps12 pre-mRNA. Plant Cell. 2006;18(10): 2650-63.

15. Tadini L, Ferrari R, Lehniger MK, Mizzotti C, Moratti F, Resentini F, et al. Trans-splicing of plastid rps 12 transcripts, mediated by AtPPR4, is essential for embryo patterning in Arabidopsis thaliana. Planta. 2018;248(1):257-65.

16. De Longevialle AF, Hendrickson L, Taylor NL, Delannoy E, Lurin C, Badger M, et al. The pentatricopeptide repeat gene OTP51 with two LAGLIDADG motifs is required for the cis-splicing of plastid ycf3 intron 2 in Arabidopsis thaliana. Plant J. 2008;56(1):157-68.

17. Chateigner-Boutin AL, Des Francs-Small CC, Delannoy E, Kahlau S, Tanz SK, De Longevialle AF, et al. OTP70 is a pentatricopeptide repeat protein of the E subgroup involved in splicing of the plastid transcript rpoC1. Plant J. 2011; 65(4):532-42.

18. Wang Z, LV J, Xie S, Zhang Y, Qiu Z, Chen P, et al. OsSLA4 encodes a pentatricopeptide repeat protein essential for early chloroplast development and seedling growth in rice. Plant Growth Regul. 2018;84:249-60.

19. Chen L, Huang L, Dai L, Gao Y, Zou W, Lu X, et al. PALE-GREEN LEAF12 encodes a novel Pentatricopeptide repeat protein required for chloroplast development and 16S rRNA processing in Rice. Plant Cell Physiol. 2019; 60(3):587-98.

20. Beick S, Schmitz-Linneweber C, Williams-Carrier R, Jensen B, Barkan A. The pentatricopeptide repeat protein PPR5 stabilizes a specific tRNA precursor in maize chloroplasts. Mol Cell Biol. 2008;28(17):5337-47.

21. Wang $Y$, Ren $Y$, Zhou K, Liu L, Wang J, Xu Y, et al. WHITE STRIPE LEAF4 encodes a novel P-type PPR protein required for chloroplast biogenesis during early LEAF development. Front Plant Sci. 2017:8:1116.

22. Ostersetzer O, Cooke AM, Watkins KP, Barkan A. CRS1, a chloroplast group II intron splicing factor, promotes intron folding through specific interactions with two intron domains. Plant Cell. 2005;17(1):241-55.

23. Ostheimer GJ, Rojas M, Hadjivassiliou H, Barkan A. Formation of the CRS2CAF2 group II intron splicing complex is mediated by a 22-amino acid motif in the $\mathrm{COOH}$-terminal region of CAF2. J Biol Chem. 2006;281(8):47328.

24. Ostheimer GJ, Williams-Carrier R, Belcher S, Osborne E, Gierke J, Barkan A. Group II intron splicing factors derived by diversification of an ancient RNAbinding domain. EMBO J. 2003;22(15):3919-29.

25. Asakura Y, Barkan A. Arabidopsis orthologs of maize chloroplast splicing factors promote splicing of orthologous and species-specific group II introns. Plant Physiol. 2006;142(4):1656-63.

26. Asakura Y, Bayraktar OA, Barkan A. Two CRM protein subfamilies cooperate in the splicing of group IIB introns in chloroplasts. Rna. 2008;14(11):2319-32.

27. Zhang Q, Shen L, Wang Z, Hu G, Ren D, Hu J, et al. OsCAF1, a CRM domain containing protein, influences chloroplast development. Int J Mol Sci. 2019; 20:18.

28. Zhang Z, Cui X, Wang Y, Wu J, Gu X, Lu T. The RNA editing factor WSP1 is essential for chloroplast development in Rice. Mol Plant. 2017;10(1):86-98.

29. Lin D, Gong $X$, Jiang $Q$, Zheng $K$, Zhou $H$, Xu J, et al. The rice ALS3 encoding a novel pentatricopeptide repeat protein is required for chloroplast development and seedling growth. Rice (New York, NY). 2015;8:17.

30. Wang D, Liu H, Zhai G, Wang L, Shao J, Tao Y. OspTAC2 encodes a pentatricopeptide repeat protein and regulates rice chloroplast development. J Genet Genomics. 2016;43(10):601-8.

31. Jenkins BD, Kulhanek DJ, Barkan A. Nuclear mutations that block group || RNA splicing in maize chloroplasts reveal several intron classes with distinct requirements for splicing factors. Plant Cell. 1997;9(3):283-96.

32. Jenkins BD, Barkan A. Recruitment of a peptidyl-tRNA hydrolase as a facilitator of group II intron splicing in chloroplasts. EMBO J. 2001;20(4): 872-9.

33. Panicot M, Minguet EG, Ferrando A, Alcázar R, Blázquez MA, Carbonell J, et al. A polyamine metabolon involving aminopropyl transferase complexes in Arabidopsis. Plant Cell. 2002;14(10):2539-51.

34. Wang C, Shen L, Fu Y, Yan C, Wang K. A simple CRISPR/Cas9 system for multiplex genome editing in Rice. J Genet Genom. 2015;42(12):703-6.

35. Ma X, Chen L, Zhu Q, Chen Y, Liu Y. Rapid decoding of sequence-specific nuclease-induced heterozygous and Biallelic mutations by direct sequencing of PCR products. Mol Plant. 2015;8(8):1285-7.

36. Lichtenthaler H. Chlorophylls and carotenoids: pigments of photosynthetic biomembranes. Methods Enzymol. 1987;148:350-82.
37. Livak KJ, Schmittgen TD. Analysis of relative gene expression data using real-time quantitative PCR and the $2^{-\Delta \Delta C T}$ method. Methods. 2001;25(4): 402-8.

38. Zhang Y, Su J, Duan S, Ao Y, Dai J, Liu J, et al. A highly efficient rice green tissue protoplast system for transient gene expression and studying light/ chloroplast-related processes. Plant Methods. 2011;7(1):30.

\section{Publisher's Note}

Springer Nature remains neutral with regard to jurisdictional claims in published maps and institutional affiliations.
Ready to submit your research? Choose BMC and benefit from:

- fast, convenient online submission

- thorough peer review by experienced researchers in your field

- rapid publication on acceptance

- support for research data, including large and complex data types

- gold Open Access which fosters wider collaboration and increased citations

- maximum visibility for your research: over $100 \mathrm{M}$ website views per year

At BMC, research is always in progress.

Learn more biomedcentral.com/submissions 